\title{
Bose and Mott glass phases in dimerized quantum antiferromagnets
}

\author{
S. J. Thomson ${ }^{1,2}$ and F. Krüger ${ }^{2,3}$ \\ ${ }^{1}$ SUPA, School of Physics and Astronomy, University of St. Andrews, North Haugh, St. Andrews KY16 9SS, United Kingdom \\ ${ }^{2}$ ISIS Facility, Rutherford Appleton Laboratory, Chilton, Didcot, Oxfordshire OX11 0QX, United Kingdom \\ ${ }^{3}$ London Centre for Nanotechnology, University College London, Gordon Street, London WC1H OAH, United Kingdom
}

(Received 29 June 2015; published 4 November 2015)

\begin{abstract}
We examine the effects of disorder on dimerized quantum antiferromagnets in a magnetic field, using the mapping to a lattice gas of hard-core bosons with finite-range interactions. Combining a strong-coupling expansion, the replica method, and a one-loop renormalization-group analysis, we investigate the nature of the glass phases formed. We find that away from the tips of the Mott lobes, the transition is from a Mott insulator to a compressible Bose glass, however the compressibility at the tips is strongly suppressed. We identify this finding with the presence of a rare Mott glass phase and demonstrate that the inclusion of replica symmetry breaking is vital to correctly describe the glassy phases. This result suggests that the formation of Bose and Mott glass phases is not simply a weak localization phenomenon but is indicative of much richer physics. We discuss our results in the context of both ultracold atomic gases and spin-dimer materials.
\end{abstract}

DOI: 10.1103/PhysRevB.92.180201

The disordered Bose-Hubbard model is an ideal system for the thorough study of the effects of disorder on strongly interacting quantum systems. Ultracold atoms in optical lattices [1-5] perhaps offer the most direct experimental system in which to realize Bose-Hubbard physics, however the small system sizes and destructive nature of many measurements limit the efficacy of experiments. Dimerized quantum antiferromagnets present a compelling alternative environment due to an exact mapping to a lattice gas of bosons with hard-core repulsion [6-8]. These systems consist of lattices of pairs of spins (dimers) which, in the ground state, are all in a singlet configuration. This state can be viewed as an "empty" lattice while a local triplet excitation can be thought of as a site occupied by a spin-1 boson ("triplon").

Condensation of these bosons corresponds to exotic magnetically ordered states seen in materials such as $\mathrm{TlCuCl}_{3}$ [9-11], $\mathrm{Cs}_{2} \mathrm{CuCl}_{4}$ [12,13], $\mathrm{BaCuSi}_{2} \mathrm{O}_{6}$ [14,15], $\mathrm{SrCu}_{2}\left(\mathrm{BO}_{3}\right)_{2}$ [16,17], and $\mathrm{Ba}_{3} \mathrm{Mn}_{2} \mathrm{O}_{8}$ [18-20]. These systems provide excellent experimental setups to probe quantum critical behavior through field and pressure tuning, and have motivated some notable theoretical works based on bondoperator techniques [21-24].

Recent experiments on disordered quantum antiferromagnets have seen evidence for interesting glassy phases, particularly in bromine-doped dichloro-tetrakis-thiourea-nickel (DTN) [25] where both Bose and Mott glass phases of bosonic quasiparticles have been observed. Such phases have also been seen in other materials [26-29] and in quantum Monte Carlo simulations [30-34].

Motivated by these experimements, in this Rapid Communication we present an analytic treatment of dimerized quantum antiferromagnets with weak intradimer bond disorder using the hard-core boson formalism. We perform a strong-coupling expansion $[35,36]$ combined with a replica disorder average to derive an effective field theory. From a renormalization-group (RG) analysis we obtain the phase boundaries between the gapped magnetic states-or in boson language, incompressible Mott insulating states-and the adjacent spin-glass phases. We show that away from the tips of the Mott lobes, the spin glass is equivalent to a compressible
PACS number(s): 75.10.Jm, 05.30.Jp, 64.60.ae, 64.70.Tg

Bose glass, while at the tips we have strong indication for the existence of an incompressible Mott glass.

The finite compressibility of the Bose glass turns out to be a direct consequence of replica symmetry breaking (RSB), a mathematical property signifying the nonergodic nature of the glassy states. Our work clearly shows that RSB in disordered Bose-Hubbard models is directly linked to the physical properties of the glassy phases and that it finds a natural interpretation in terms of analogous disordered spin systems.

We start from a Hamiltonian describing a lattice of coupled dimers of $S=1 / 2$ spins, subject to single-dimer anisotropy $D$ [37] and magnetic field $h$,

$$
\begin{aligned}
\mathcal{H}= & \sum_{i}\left[J_{0} \hat{\mathbf{S}}_{i 1} \cdot \hat{\mathbf{S}}_{i 2}-D\left(\hat{S}_{i 1}^{z}+\hat{S}_{i 2}^{z}\right)^{2}-h\left(\hat{S}_{i 1}^{z}+\hat{S}_{i 2}^{z}\right)\right] \\
& +\frac{1}{2} \sum_{i \neq j} \sum_{m, n} J_{i j m n} \hat{\mathbf{S}}_{i m} \cdot \hat{\mathbf{S}}_{j n},
\end{aligned}
$$

where $i, j$ label the dimers and $m, n=1,2$ the component spins of the dimers. This Hamiltonian is quite generic and describes most of the aforementioned dimer compounds [9-17,21]. For simplicity, we assume that the dimers are located on the sites of a $d$-dimensional hypercubic lattice and that the couplings between dimers are isotropic along different bond directions. To be specific, we consider superexchanges $J_{1}$ and $J_{2}$ between adjacent dimers (Fig. 1), where $J_{0} \gg J_{1}>J_{2}>0$.

The mapping of the dimerized quantum antiferromagnet to a model of hard-core bosons is achieved by expressing the spin operators of each dimer in terms of singlet and triplet bond operators [21-24], $\hat{s}^{\dagger}|0\rangle=\frac{1}{\sqrt{2}}(|\uparrow \downarrow\rangle-|\downarrow \uparrow\rangle), \hat{t}_{+}^{\dagger}|0\rangle=$ $|\uparrow \uparrow\rangle, \hat{t}_{0}^{\dagger}|0\rangle=\frac{1}{\sqrt{2}}(|\uparrow \downarrow\rangle+|\downarrow \uparrow\rangle)$, and $\hat{t}_{-}^{\dagger}|0\rangle=|\downarrow \downarrow\rangle$. Using the hard-core constraint $\hat{s}^{\dagger} \hat{s}+\hat{t}_{\alpha}^{\dagger} \hat{t}_{\alpha}=1$ we obtain

$$
\begin{aligned}
\mathcal{H}= & -\sum_{i, \sigma} \mu_{\sigma} \hat{n}_{i \sigma}+V \sum_{\langle i, j\rangle} \sum_{\sigma_{1}, \sigma_{2}} \sigma_{1} \sigma_{2} \hat{n}_{i \sigma_{1}} \hat{n}_{j \sigma_{2}} \\
& +t \sum_{\langle i, j\rangle}\left[\left(\hat{t}_{i-}^{\dagger}-\hat{t}_{i+}\right)\left(\hat{t}_{j-}-\hat{t}_{j+}^{\dagger}\right)+\text { H.c. }\right]
\end{aligned}
$$




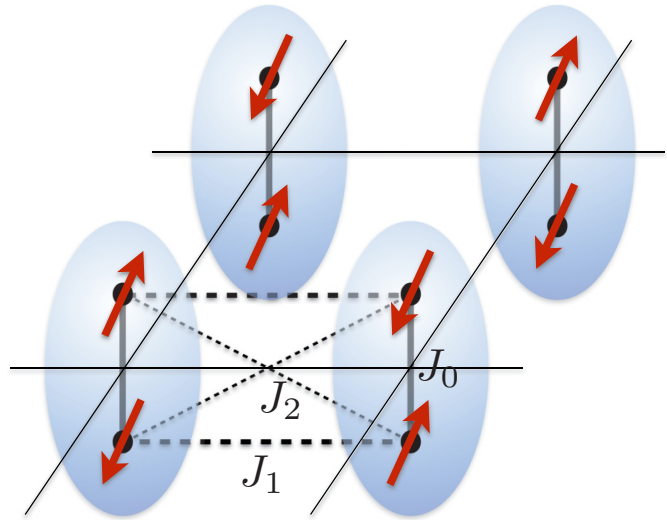

FIG. 1. (Color online) Square lattice of dimers with intradimer coupling $J_{0}$. Adjacent dimers are coupled by exchanges $J_{1}$ and $J_{2}$.

with $\hat{n}_{i \sigma}=\hat{t}_{i \sigma}^{\dagger} \hat{t}_{i \sigma}, \quad t=\left(J_{1}-J_{2}\right) / 2, \quad V=\left(J_{1}+J_{2}\right) / 2$, and $\mu_{\sigma}=-\left(J_{0}-D\right)+\sigma h$. The inclusion of an anisotropy $D>0$ is not crucial for our analysis but simplifies matters by allowing us to project out the $t_{0}$ triplet which is energetically unfavorable.

The different Mott insulating states can be easily found in the atomic limit $t \rightarrow 0$. For $|h|<J_{0}-D$, the occupation numbers of both triplets are zero, corresponding to a gapped nonmagnetic state. For sufficiently strong fields the magnetization is fully saturated with exactly one triplon on every site, $m_{+}=1$ or $m_{-}=1$, depending on the sign of $h$. We label these states as $m= \pm 1$ Mott insulators, respectively. Between the nonmagnetic and fully polarized states, repulsive interactions between triplons on neighboring sites stabilize checkerboard order where every second site remains empty ( $m= \pm 1 / 2$ ). Dimer couplings beyond nearest neighbors lead to additional incommensurate states with filling fractions that crucially depend on the lattice geometry. In all Mott insulating phases, the magnetization does not change as a function of field, giving rise to magnetization plateaus.

For large enough $t$, the system becomes a superfluid, corresponding to a canted $X Y$ antiferromagnet. The phase boundaries are obtained from a strong-coupling expansion around the atomic limit. Performing a Hubbard-Stratonovich decoupling of the hopping term, we obtain a dual continuum action for the superfluid order parameter $\psi(\mathbf{r}, \tau)$ in space and imaginary time [38-40],

$$
\mathcal{S}_{0}=\int_{\mathbf{k} \omega} K(\mathbf{k}, \omega)|\psi(\mathbf{k}, \omega)|^{2}+u \int_{\mathbf{r} \tau}|\psi(\mathbf{r}, \tau)|^{4}
$$

with $K(\mathbf{k}, \omega)=\left(k^{2}-i \gamma_{1} \omega+\gamma_{2} \omega^{2}+r\right)$ in the momentum and frequency domain. The mass $r$ and the interaction vertex $u$ are related to the local single- and two-particle Green functions for the different Mott insulating states respectively, e.g., $r=$ $r_{m}=1+2 t d G_{m}(\omega=0)$ [41]. The MI/SF mean-field phase boundaries are obtained by $r=0$ and shown as dashed lines in Fig. 2 as a function of dimensionless dimer coupling $x$ and magnetic field $y$. The phase diagram has the familiar lobe structure of Bose-Hubbard models and is symmetric around $h=0$. It has been suggested [42-44] that the tips of the fractionally filled lobes may exhibit first-order or supersolid

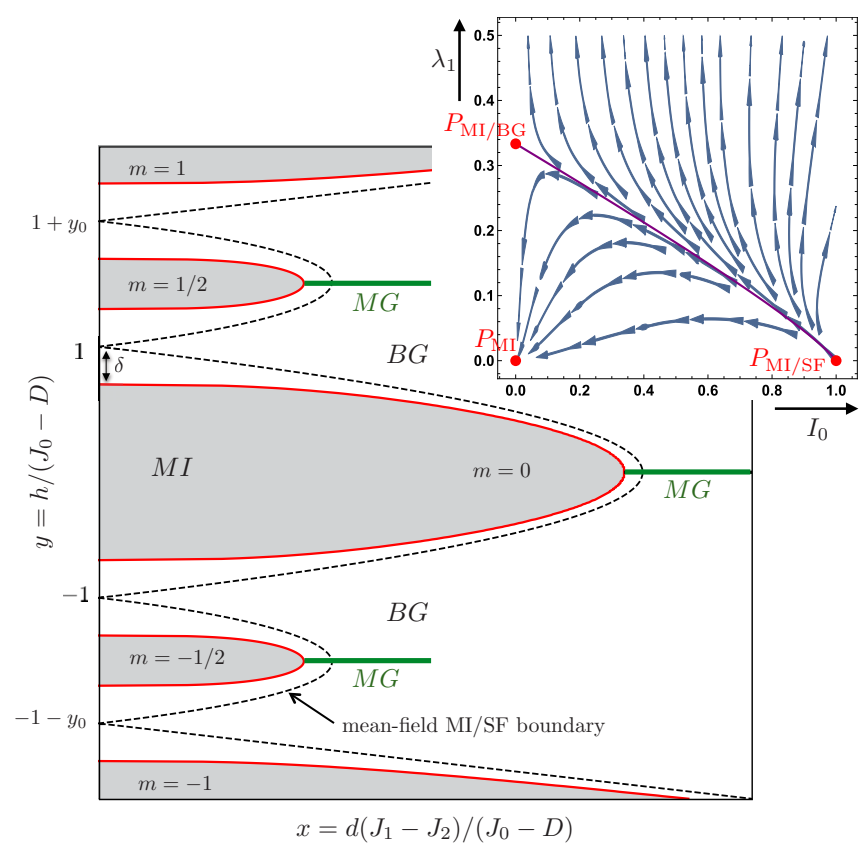

FIG. 2. (Color online) Phase diagram as a function of dimer coupling $x$ and magnetic field $y$ for $y_{0}=d\left(J_{1}+J_{2}\right) /\left(J_{0}-D\right)=1$. Dashed lines show the Mott insulator/superfluid (MI/SF) mean-field transitions of the clean system. Disorder leads to the formation of an incompressible Bose glass (BG) between the MI and the SF, which turns into an incompressible Mott glass (MG) at the tips of the Mott lobes. Solid red lines are the MI/BG phase boundaries obtained from an RG analysis in $d=3$ for a disorder strength of $\delta=\Delta /\left(J_{0}-D\right)=$ 0.3 . Inset: RG flow of the inverse mean $I_{0}=1 /(1+\bar{r})$, and relative variance $\lambda_{1} \sim\left(\overline{r^{2}}-\bar{r}^{2}\right) / \bar{r}^{2}$ of the random-mass distribution. There are three fixed points: a stable MI fixed point at $\left(I_{0}, \lambda_{1}\right)=(0,0)$, a MI/SF transition at $(1,0)$, and a MI/BG transition at $(0, d / 9)$. The $\mathrm{BG} / \mathrm{SF}$ transition is not accessible in our strong-coupling approach.

behavior, however such a question is beyond the reach of the present analysis.

The coefficients of the frequency terms are given by derivatives of the mass coefficient with respect to the magnetic field, $\gamma_{1}=-\partial r / \partial y$ and $\gamma_{2}=-\frac{1}{2} \partial^{2} r / \partial y^{2}$ [45]. At the tips of the Mott lobes, the slope $\gamma_{1}$ vanishes and the field theory becomes relativistic, reflecting the particle-hole symmetry at these points. Previous works [38-40] studying other aspects of the model have largely neglected the frequency terms, however we retain them here to study how the behavior changes near the tips. These frequency terms will turn out to be crucial to correctly describe the thermodynamics.

The key question we are trying to answer in the present work is whether the glassy phase formed in disordered BoseHubbard models is always a compressible Bose glass [46], or if the more elusive incompressible Mott glass may exist at the high-symmetry tips of the Mott lobes [30,31,33,34]. First seen in one-dimensional (1D) fermion systems [47,48], the Mott glass has also been predicted to exist in the $\mathrm{O}(2)$ quantum rotor model [49-52], which maps to the Bose-Hubbard model at commensurate fillings, and has been experimentally observed in the disordered quantum antiferromagnet DTN [25].

We focus on random mass disorder such that $\mu_{i, \sigma}=$ $\mu_{\sigma}+\varepsilon_{i}$. This can come from disorder in the intradimer 
coupling $J_{0}$, the anisotropy $D$, or the applied field $h$. In the following, we assume that the disorder has a symmetric box distribution of width $2 \Delta$ and is uncorrelated between different sites. For sufficiently bounded disorder the phase diagram retains Mott insulating regions, e.g., a central Mott lobe is present for $\delta=\Delta /\left(J_{0}-D\right)<1$. The Hubbard-Stratonovich transformation is performed in the same way, leading to disorder in all coefficients of the dual action. We use the replica trick [53] to obtain the disorder averaged free energy. The replicated action

$$
\mathcal{S}=\sum_{\alpha=1}^{n} \overline{\mathcal{S}_{0}\left[\psi_{\alpha}^{*}, \psi_{\alpha}\right]}-\frac{g}{2} \sum_{\alpha \beta} \int_{\mathbf{r} \tau \tau^{\prime}}\left|\psi_{\alpha}(\mathbf{r}, \tau)\right|^{2}\left|\psi_{\beta}\left(\mathbf{r}, \tau^{\prime}\right)\right|^{2}
$$

consists of two parts. The first contribution is simply $n$ copies of the original action with disorder averaged coefficients $\bar{\gamma}_{1}$, $\bar{\gamma}_{2}, \bar{r}$, and $\bar{u}$. The second term is the disorder vertex, which is nondiagonal in replica space $\alpha, \beta$ and imaginary time and proportional to the variance of the random-mass distribution $g=\left(\overline{r^{2}}-\bar{r}^{2}\right)$.

To determine the phase diagram in the presence of weak disorder, we use a momentum-shell RG approach. As in previous work [38-40], we make the change of variables $I_{0}=1 /(1+\bar{r})$, and introduce the relative disorder variance $\lambda=I_{0}^{2} g$ to distinguish between the Mott insulating and glassy phases. In all but the superfluid phase, $I_{0}$ flows to zero, reflecting the short-ranged superfluid correlations. The relative variance $\lambda$ compares the shift of the random mass distribution with its spread. In the Mott insulator, the distribution shifts faster than it spreads and $\lambda$ renormalizes to zero. If the spread is faster than the shift, the tail of the distribution pushes through zero, indicating a glassy phase where the physics is dominated by rare superfluid regions. Taking into account the one-step RSB in this model [40], the RG equations are

$$
\begin{aligned}
I_{0}^{\prime}(\ell) & =\left(3 / 2 \lambda_{1}-2\right) I_{0}+2 I_{0}^{2}, \\
\lambda_{1}^{\prime}(\ell) & =\left(4 I_{0}-d\right) \lambda_{1}+9 \lambda_{1}^{2}, \\
\bar{\gamma}_{1}^{\prime}(\ell) & =\left(2-z+\lambda_{1}\right) \bar{\gamma}_{1}, \\
\bar{\gamma}_{2}^{\prime}(\ell) & =\left(2-2 z+\lambda_{1}\right) \bar{\gamma}_{2}+\lambda_{1} I_{0} \bar{\gamma}_{1}^{2},
\end{aligned}
$$

where $z$ is the dynamical critical exponent and $\lambda_{0} \equiv 0$ and $\lambda_{1}=I_{0}^{2} g_{1}$ denote the step heights of the Parisi disorder function $[41,53]$. We neglect the $\bar{u}$ vertex since it is irrelevant away from the tips $\left(\bar{\gamma}_{1} \neq 0\right)$. The RG flow in the $I_{0}-\lambda_{1}$ plane is shown in the inset in Fig. 2. The MI/SF fixed point is unstable against disorder, confirming that even for infinitesimal disorder, the transition from the Mott insulator is into a disordered insulating state and not into a superfluid [54,55]. As we show later, the disordered state is a compressible Bose glass, except for the tips where we see strong indications for an incompressible Mott glass state.

The relative variance $\lambda_{1}$ diverges in the Bose glass. We stop integration at a scale $\ell^{*}$ where $\lambda_{1}\left(\ell^{*}\right)=1$ and our RG becomes invalid. This scale can be identified with a correlation length $\xi \sim e^{\ell^{*}}$ which corresponds to the typical separation of superfluid regions. Note that $\xi$ is not the superfluid correlation length, which remains finite at the MI/BG transition. Linearizing near $P_{\mathrm{MI} / \mathrm{BG}}$, we find the correlation length diverges as $\xi \sim\left(x-x_{c}\right)^{-1 / d}$.
From the dependence of $I_{0}$ and $\lambda_{1}$ on the microscopic parameters, we can determine the MI/BG phase boundary as a function of the dimensionless dimer coupling $x$ and magnetic field $y$ for a given disorder strength $\delta$ (Fig. 2). As in the conventional Bose-Hubbard model, the Mott insulating regions shrink when disorder is added, and a glassy phase intervenes between the Mott insulating and the superfluid regions. In the original magnetic language, this corresponds to a spin-glass phase.

To determine the nature of the glassy phase, we calculate the compressibility. For a single-component boson system, the local compressibility is defined as the derivative of the local density with respect to the chemical potential and related to the particle-number fluctuations, $\kappa_{i}=\partial\left\langle\hat{n}_{i}\right\rangle / \partial \mu=$ $\beta \sum_{j}\left(\left\langle\hat{n}_{i} \hat{n}_{j}\right\rangle-\left\langle\hat{n}_{j}\right\rangle\left\langle\hat{n}_{j}\right\rangle\right)$. This is easily generalized to the two-triplon case, where the compressibility is defined as the derivative of the local magnetization with respect to magnetic field. Performing a disorder average we can calculate $\kappa=$ $-\partial^{2} \overline{\mathcal{F}} / \partial y^{2}$ directly from the replica field theory, yielding [41]

$$
\kappa=\frac{2 S_{d}}{(2 \pi)^{d}} \frac{I_{0}^{2} \bar{\gamma}_{2}^{2} \lambda_{1}}{\left(I_{0}^{2} \bar{\gamma}_{1}^{2}+4 I_{0} \bar{\gamma}_{2}\right)^{3 / 2}},
$$

where $S_{d}$ is the surface of a $d$-dimensional unit sphere. It is crucial here to include the effects of one-step RSB,
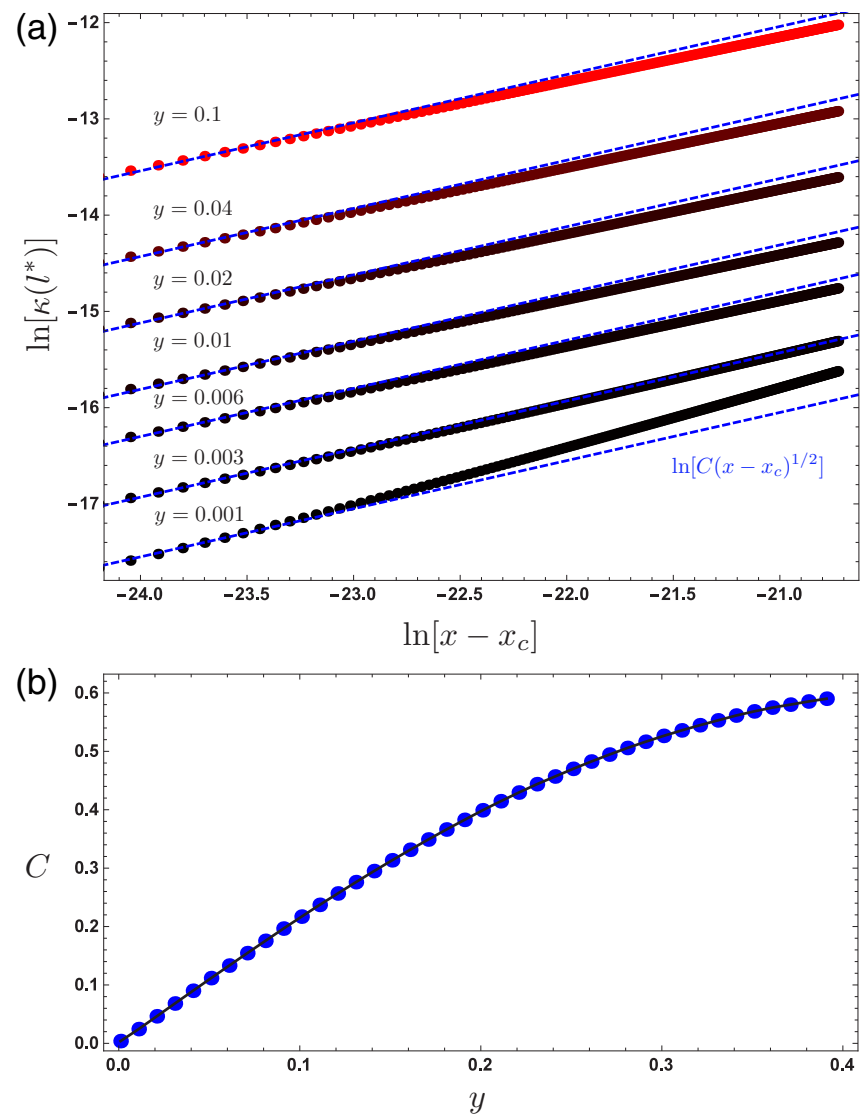

FIG. 3. (Color online) (a) Power-law behavior of the compressibility $\kappa\left(l^{*}\right) \simeq C\left(x-x_{c}\right)^{1 / 2}(d=3)$ of the Bose glass close to the transition to the Mott insulator. The blue dashed lines are guides to the eye with gradient $1 / 2$. Near $y=0$ there is an anomalous suppression of the range of universal behavior. (b) $C$ vs $y$, showing the vanishing compressibility close to the tip of the central lobe at $y=0$. 
otherwise $\kappa$ vanishes identically as a consequence of the frequency structure that is inherent to all Bose-Hubbard models $[41,45]$. This remarkable result reveals the surprising physical importance of RSB in this system. Linearizing around $P_{\mathrm{MI} / \mathrm{Bg}}$ we find that at the transition to the Mott insulator the compressibility vanishes as

$$
\kappa=\kappa\left(\ell^{*}\right)=C\left(x-x_{c}\right)^{2 / d-1 / 6},
$$

where $C \sim\left|\bar{\gamma}_{1}\right|$. Away from the tip, the disordered state adjacent to the Mott insulator is a compressible Bose glass. Approaching a particle-hole symmetric Mott lobe tip, the coefficient $C$ vanishes, suggesting a change of universality. The analytical result (7) is confirmed by a numerical calculation of $\kappa\left(\ell^{*}\right)$ from the full RG equations (5) for the central Mott lobe in $d=3$ with $\delta=0.3$ [see Fig. 3(a)]. The coefficient $C$ has a strong dependence on the field and vanishes linearly as $y \rightarrow 0$ [Fig. 3(b)]. Very close to the tip at $y=0$, there is a strong suppression of the range of universal behavior, but no indication of a crossover to a different universality class. This highlights the singular nature of this point and strongly indicates the existence of an incompressible Mott glass state.

Although we have focused on dimerized quantum antiferromagnets using the hard-core boson formalism, our prediction of a Mott glass is valid across a wide range of systems, including conventional Bose-Hubbard and Jaynes-Cummings Hubbard [56] models. Any such Bose-Hubbard-like model may be treated using the methods outlined here, with the microscopic differences appearing only in the UV-scale starting values of the flow parameters. This prediction lends weight to previous numerical quantum Monte Carlo work [31,34]. It may also explain the controversy over the existence of a direct MI/SF transition at the tips of the Mott lobes in the disordered Bose-Hubbard model: previous works which used compressibility as the criterion for the onset of a glassy phase will necessarily have missed the transition between the Mott insulator and the Mott glass.

The breakdown of self-averaging [39,57,58], the importance of replica symmetry breaking [40], and the connection with spin-glass phenomena strongly suggest that the formation of Bose and Mott glass phases is not simply a weak localization phenomenon. It would be interesting to review the nature of these phases in the context of many-body localization [59] and the related entanglement entropy scaling [60].

The equivalence between Bose-Hubbard models and dimerized quantum antiferromagnets allows for multiple complementary experiments to verify our theoretical predictions. Previous measurements on disordered ultracold atomic gases have inferred the presence of the Bose glass from macroscopic measurements [61-63], however the boson number fluctuations associated with the local compressibility are also now within reach of quantum gas microscope systems [4,5], potentially allowing for direct imaging of the glassy phases. Thermodynamic measurements of the magnetic susceptibility and the specific heat of dimerized quantum antiferromagnets can provide clear signatures of Bose and Mott glass phases [25]. We also expect to see characteristic differences in the glassy dynamics, which could be studied with $\mu \mathrm{SR}$, as well as in the magnetic excitation spectra. Dimer systems exhibiting geometric frustration are a particularly intriguing theoretical problem for further study, as are additional types of disorder such as nonmagnetic impurities [20].

The authors would like to thank B. Braunecker, G. D. Bruce, C. Hooley, J. Keeling, and C. Pedder for valuable discussions. S.J.T. acknowledges financial support from EPSRC (UK) via the Scottish Doctoral Training Centre in Condensed Matter Physics.
[1] D. Jaksch, C. Bruder, J. I. Cirac, C. W. Gardiner, and P. Zoller, Phys. Rev. Lett. 81, 3108 (1998).

[2] M. Greiner, O. Mandel, T. Esslinger, T. W. Hansch, and I. Bloch, Nature (London) 415, 39 (2002).

[3] I. Bloch, Nat. Phys. 1, 23 (2005).

[4] W. S. Bakr, J. I. Gillen, A. Peng, S. Fölling, and M. Greiner, Nature (London) 462, 74 (2009).

[5] J. F. Sherson, C. Weitenberg, M. Endres, M. Cheneau, I. Bloch, and S. Kuhr, Nature (London) 467, 68 (2010).

[6] T. Matsubara and H. Matsuda, Prog. Theor. Phys. 16, 569 (1956).

[7] T. Giamarchi, C. Ruegg, and O. Tchernyshynov, Nat. Phys. 4, 198 (2008).

[8] V. Zapf, M. Jaime, and C. D. Batista, Rev. Mod. Phys. 86, 563 (2014).

[9] T. Nikuni, M. Oshikawa, A. Oosawa, and H. Tanaka, Phys. Rev. Lett. 84, 5868 (2000).

[10] C. Ruegg, N. Cavadini, A. Furrer, H.-U. Gudel, K. Kramer, H. Mutka, A. Wildes, K. Habicht, and P. Vorderwisch, Nature (London) 423, 62 (2003).

[11] P. Merchant, B. Normand, K. W. Kramer, M. Boehm, D. F. McMorrow, and C. Ruegg, Nat. Phys. 10, 373 (2014).

[12] R. Coldea, D. A. Tennant, K. Habicht, P. Smeibidl, C. Wolters, and Z. Tylczynski, Phys. Rev. Lett. 88, 137203 (2002).
[13] T. Radu, H. Wilhelm, V. Yushankhai, D. Kovrizhin, R. Coldea, Z. Tylczynski, T. Lühmann, and F. Steglich, Phys. Rev. Lett. 95, 127202 (2005).

[14] S. E. Sebastian, N. Harrison, C. D. Batista, L. Balicas, M. Jaime, P. A. Sharma, N. Kawashima, and I. R. Fisher, Nature (London) 441, 617 (2006).

[15] C. D. Batista, J. Schmalian, N. Kawashima, P. Sengupta, S. E. Sebastian, N. Harrison, M. Jaime, and I. R. Fisher, Phys. Rev. Lett. 98, 257201 (2007).

[16] K. Kodama, M. Takigawa, M. Horvati, C. Berthier, H. Kageyama, Y. Ueda, S. Miyahara, F. Becca, and F. Mila, Science 298, 395 (2002).

[17] M. Jaime, R. Daou, S. A. Crooker, F. Weickert, A. Uchida, A. E. Feiguin, C. D. Batista, H. A. Dabkowska, and B. D. Gaulin, Proc. Natl. Acad. Sci. U.S.A. 109, 12404 (2012).

[18] E. C. Samulon, K. A. Al-Hassanieh, Y.-J. Jo, M. C. Shapiro, L. Balicas, C. D. Batista, and I. R. Fisher, Phys. Rev. B 81, 104421 (2010).

[19] S. Suh, K. A. Al-Hassanieh, E. C. Samulon, I. R. Fisher, S. E. Brown, and C. D. Batista, Phys. Rev. B 84, 054413 (2011).

[20] E. C. Samulon, M. C. Shapiro, and I. R. Fisher, Phys. Rev. B 84, 054417 (2011).

[21] O. Rösch and M. Vojta, Phys. Rev. B 76, 224408 (2007). 
[22] R. L. Doretto and M. Vojta, Phys. Rev. B 85, 104416 (2012).

[23] D. G. Joshi, K. Coester, K. P. Schmidt, and M. Vojta, Phys. Rev. B 91, 094404 (2015).

[24] D. G. Joshi and M. Vojta, Phys. Rev. B 91, 094405 (2015).

[25] R. Yu, T. Liang, N. S. Sullivan, J. S. Xia, C. Huan, A. PaduanFilho, N. F. Oliveira, S. Haas, A. Steppke, C. F. Miclea et al., Nature (London) 489, 379 (2012).

[26] T. Hong, A. Zheludev, H. Manaka, and L.-P. Regnault, Phys. Rev. B 81, 060410 (2010).

[27] M. B. Stone, A. Podlesnyak, G. Ehlers, A. Huq, E. C. Samulon, M. C. Shapiro, and I. R. Fisher, J. Phys.: Condens. Matter 23, 416003 (2011).

[28] F. Yamada, H. Tanaka, T. Ono, and H. Nojiri, Phys. Rev. B 83, 020409 (2011).

[29] E. Wulf, S. Mühlbauer, T. Yankova, and A. Zheludev, Phys. Rev. B 84, 174414 (2011).

[30] T. Roscilde and S. Haas, Phys. Rev. Lett. 99, 047205 (2007).

[31] P. Sengupta and S. Haas, Phys. Rev. Lett. 99, 050403 (2007).

[32] R. Yu, O. Nohadani, S. Haas, and T. Roscilde, Phys. Rev. B 82, 134437 (2010).

[33] N. Ma, A. W. Sandvik, and D.-X. Yao, Phys. Rev. B 90, 104425 (2014).

[34] Y. Wang, W. Guo, and A. W. Sandvik, Phys. Rev. Lett. 114, 105303 (2015).

[35] J. K. Freericks and H. Monien, Phys. Rev. B 53, 2691 (1996).

[36] K. Sengupta and N. Dupuis, Phys. Rev. A 71, 033629 (2005).

[37] Note that single-ion anisotropy $\sim\left[\left(\hat{S}_{i 1}^{z}\right)^{2}+\left(\hat{S}_{i 2}^{z}\right)^{2}\right]$ is irrelevant in spin-1/2 dimer systems as it does not lift the degeneracy of the triplets. Our anisotropy $D$ is equivalent to a term $2 D \hat{S}_{i 1}^{z} \hat{S}_{i 2}^{z}$ ( $x x z$ intradimer coupling) and acts like a single-ion anisotropy for the spin-1 triplet excitations.

[38] F. Krüger, J. Wu, and P. Phillips, Phys. Rev. B 80, 094526 (2009).

[39] F. Krüger, S. Hong, and P. Phillips, Phys. Rev. B 84, 115118 (2011).

[40] S. J. Thomson and F. Krüger, Europhys. Lett. 108, 30002 (2014).

[41] See Supplemental Material at http://link.aps.org/supplemental/ 10.1103/PhysRevB.92.180201 for further details on the replica field theory and detailed derivation of the compressibility expression.

[42] G. G. Batrouni and R. T. Scalettar, Phys. Rev. Lett. 84, 1599 (2000).

[43] D. Yamamoto, A. Masaki, and I. Danshita, Phys. Rev. B 86, 054516 (2012).
[44] D. Yamamoto, T. Ozaki, C. A. R. Sá de Melo, and I. Danshita, Phys. Rev. A 88, 033624 (2013).

[45] The frequency dependence in the quadratic action of the dual field theory enters through the local Green function that is a function of $y+i \omega$, where $y$ is the dimensionless chemical potential or the equivalent magnetic field. From this it follows immediately that the frequency coefficients $\gamma_{i}$ are given by $i$ th-order derivatives of the mass coefficient $r$ with respect to $y$.

[46] M. P. A. Fisher, P. B. Weichman, G. Grinstein, and D. S. Fisher, Phys. Rev. B 40, 546 (1989).

[47] E. Orignac, T. Giamarchi, and P. Le Doussal, Phys. Rev. Lett. 83, 2378 (1999).

[48] T. Giamarchi, P. Le Doussal, and E. Orignac, Phys. Rev. B 64, 245119 (2001).

[49] E. Altman, Y. Kafri, A. Polkovnikov, and G. Refael, Phys. Rev. Lett. 93, 150402 (2004).

[50] E. Altman, Y. Kafri, A. Polkovnikov, and G. Refael, Phys. Rev. Lett. 100, 170402 (2008).

[51] E. Altman, Y. Kafri, A. Polkovnikov, and G. Refael, Phys. Rev. B 81, 174528 (2010).

[52] S. Iyer, D. Pekker, and G. Refael, Phys. Rev. B 85, 094202 (2012).

[53] M. Mezard and G. Parisi, J. Phys. I (France) 1, 809 (1991).

[54] L. Pollet, N. V. Prokof'ev, B. V. Svistunov, and M. Troyer, Phys. Rev. Lett. 103, 140402 (2009).

[55] V. Gurarie, L. Pollet, N. V. Prokof'ev, B. V. Svistunov, and M. Troyer, Phys. Rev. B 80, 214519 (2009).

[56] D. G. Angelakis, M. F. Santos, and S. Bose, Phys. Rev. A 76 , 031805 (2007).

[57] A. Hegg, F. Krüger, and P. W. Phillips, Phys. Rev. B 88, 134206 (2013).

[58] J. P. Álvarez Zúñiga, D. J. Luitz, G. Lemarié, and N. Laflorencie, Phys. Rev. Lett. 114, 155301 (2015).

[59] R. Nandkishore and D. Huse, Ann. Rev. Condens. Matter Phys. 6, 15 (2015).

[60] J. A. Kjäll, J. H. Bardarson, and F. Pollmann, Phys. Rev. Lett. 113, 107204 (2014).

[61] J. E. Lye, L. Fallani, M. Modugno, D. S. Wiersma, C. Fort, and M. Inguscio, Phys. Rev. Lett. 95, 070401 (2005).

[62] L. Fallani, J. E. Lye, V. Guarrera, C. Fort, and M. Inguscio, Phys. Rev. Lett. 98, 130404 (2007).

[63] C. Meldgin, U. Ray, P. Russ, D. Ceperley, and B. DeMarco, arXiv:1502.02333. 